\title{
Options for gain elements and gas mixtures in a high rate EIC Time Projection Chamber
}

\section{CPAD 2021 \\ Virtual Event @ Stony Brook University, March 18-22, 2021}

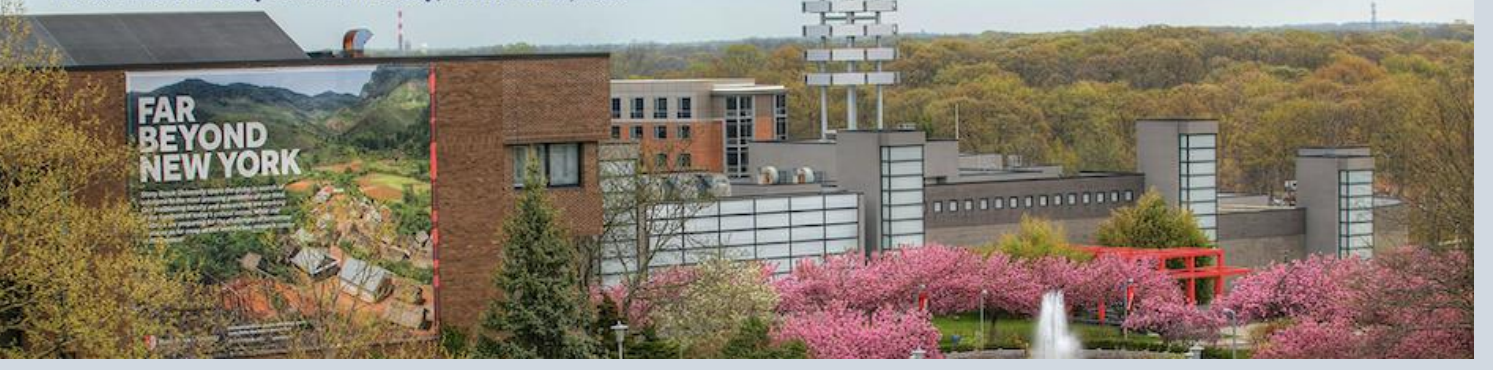

BOB AZMOUN ${ }^{1}$, CAITLIN BEATTIE ${ }^{2}$, JOHN W. HARRIS ${ }^{2}$

ALEXANDER KISELEV ${ }^{1}$, RICHARD MAJKA ${ }^{3}$, NIKOLAI SMIRNOV ${ }^{2}$, CRAIG WOODY ${ }^{1}$

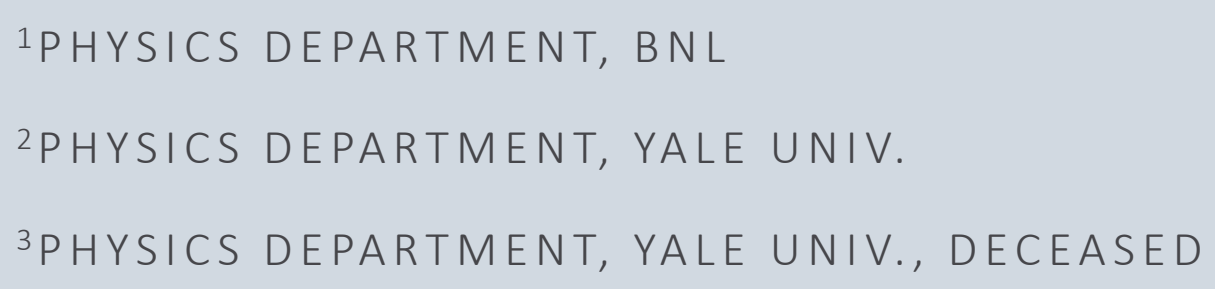


$>$ A Time Projection Chamber (TPC) is a good choice for central tracking at EIC

- Low mass, large-continuous active volume

- Precision tracking

- Pattern recognition

- Momentum reconstruction

- Particle identification

\section{- Requirements:}

- Operate at and acquire data for collision rates as high as $500 \mathrm{kHz}$

- Continuous readout option should be considered (active gating grid is not possible, but perhaps a passive (bi-polar) one is)

- Operate in magnetic field in the range $1.5-3 \mathrm{~T}$

- Limited space: $r \sim 80 \mathrm{~cm}, \mathrm{z} \sim+/-100 \mathrm{~cm}$ inside a SC solenoidal magnet.

- Main challenge: Space Charge Distortions of the E-field will be the main degradation factor for tracking and $\mathrm{dE} / \mathrm{dx}$ performance (due to high interaction rate at ultimate EIC luminosity, possibly high backgrounds, and high demands on precision)

- Crucial EIC TPC parameters : ion back flow (IBF), energy resolution (dE/dX), electron and ion drift speed, electron diffusion (in E- and B- fields), gain structure, and stability

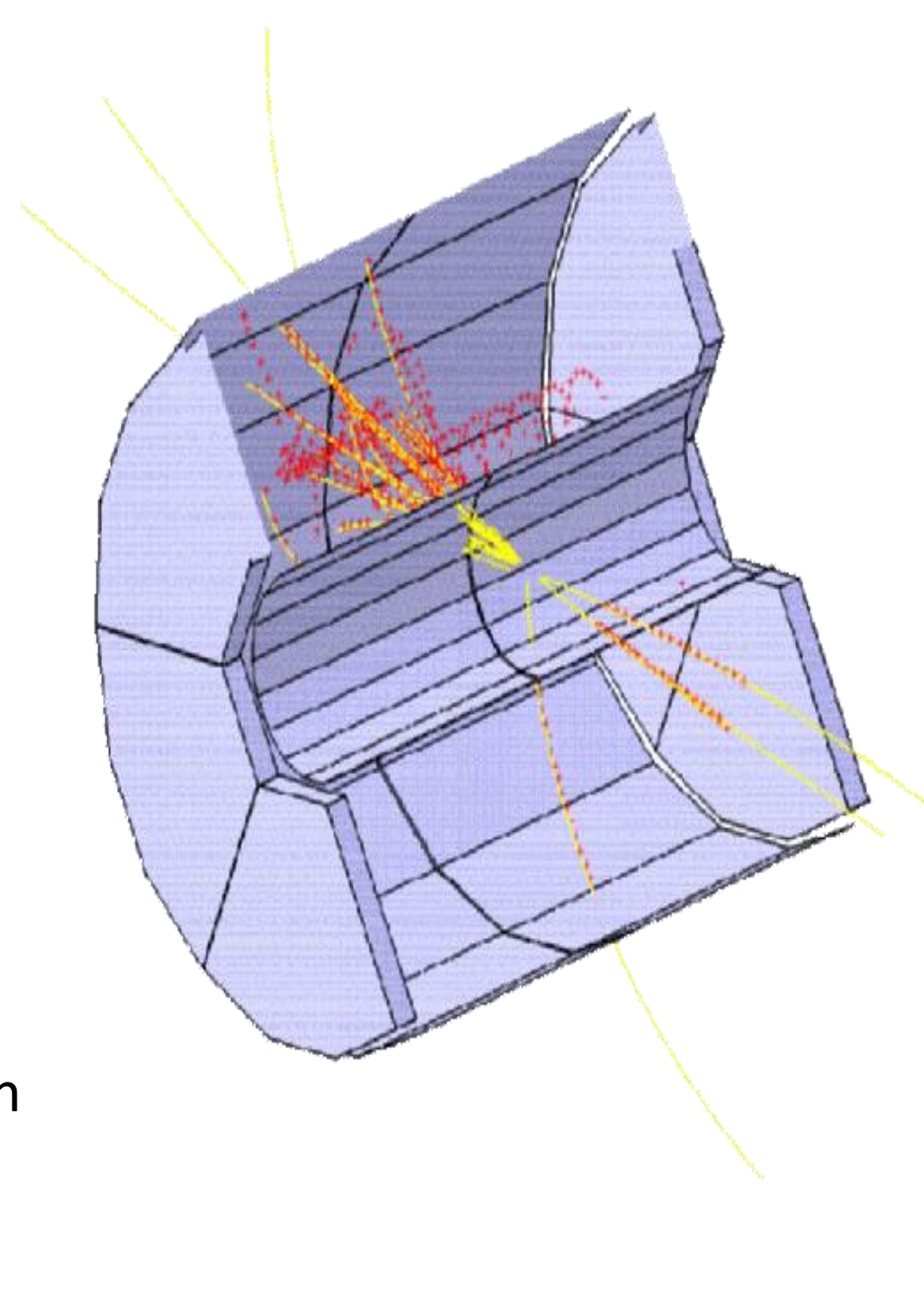




\section{EIC TPC Gas Mixture Options}

$>$ One TPC gas option that satisfies most criteria is a mixture of $\mathrm{Ne}+\mathrm{CF}_{4}$

- SPHENIX TPC selected this mixture: low diffusion (for high spatial resolution) and high ion mobility (Ar provides greater primary ionization and may be preferable in terms of energy resolution)

Three issues:

- Strong $\mathrm{CF}_{4}$ electron absorption resonance

- Possible transfer of ion charge: $\mathrm{Ne}^{+}+\mathrm{CF}_{4} \rightarrow \mathrm{Ne}+\mathrm{CF}_{3}{ }^{+}+\mathrm{F} \quad[1]$

- Both components are transparent to UV, and can be a source of scintillation causing photon feedback

Solution:

- Add a "cool" gas component with an energy of ionization less than that for $\mathrm{CF}_{4}[2]$

- Add methane $\left(\mathrm{CH}_{4}\right)$ component

- permits transfers of positive charge from the $\mathrm{Ne}^{+}$ions to $\mathrm{CH}_{4}^{+}$

- does not degrade diffusion, or the electron and ion drift velocities

- known to be an effective quencher to stabilize gain process

- for safety reasons the level of $\mathrm{CH}_{4}$ is generally kept at or below $10 \%$

[1] D.J.G. Marque et al, "Experimental ion mobility measurements in Ne-CF4," 2019 JINST 14 P04015.

[2] J. Va'vra, "Wire Chamber Gases," SLAC-PUB-5793. 


\section{Percentage of $\mathrm{CF}_{4}$}

Initial R\&D question: what percentage of $\mathrm{CF}_{4}$ used in a $\mathrm{Ne}+$ $\mathrm{CH}_{4}(10 \%)$ gas mixture?

- GARFIELD simulation results to consider [3]:

- $\mathrm{CF}_{4}$ percentages have a small impact on transverse diffusion and electron drift speed

- Though, ion mobility increases by a factor 2 for gas mixtures with $10 \% \mathrm{CF}_{4}$ compared to $50 \%$

- Electron absorption probability decreases with increasing $\mathrm{CF}_{4}$ percentage (ie, better energy resolution at higher \%)

- The magnitude of the electric field should be increased to maintain the same gas gain with increasing $\mathrm{CF}_{4}$ percentages (ie, lower gain at higher \%)

- Thus, the gas gain (Townsend coefficient) and energy resolution (electron attachment probability) are the main trade-off
GARFIRLD: $\mathrm{Ne}+\mathrm{CF} 4(5-50 \%)+\mathrm{CH} 4(10 \%), \mathrm{B}$ field $\left.=1.5 \mathrm{~T}^{* * *}\right)$
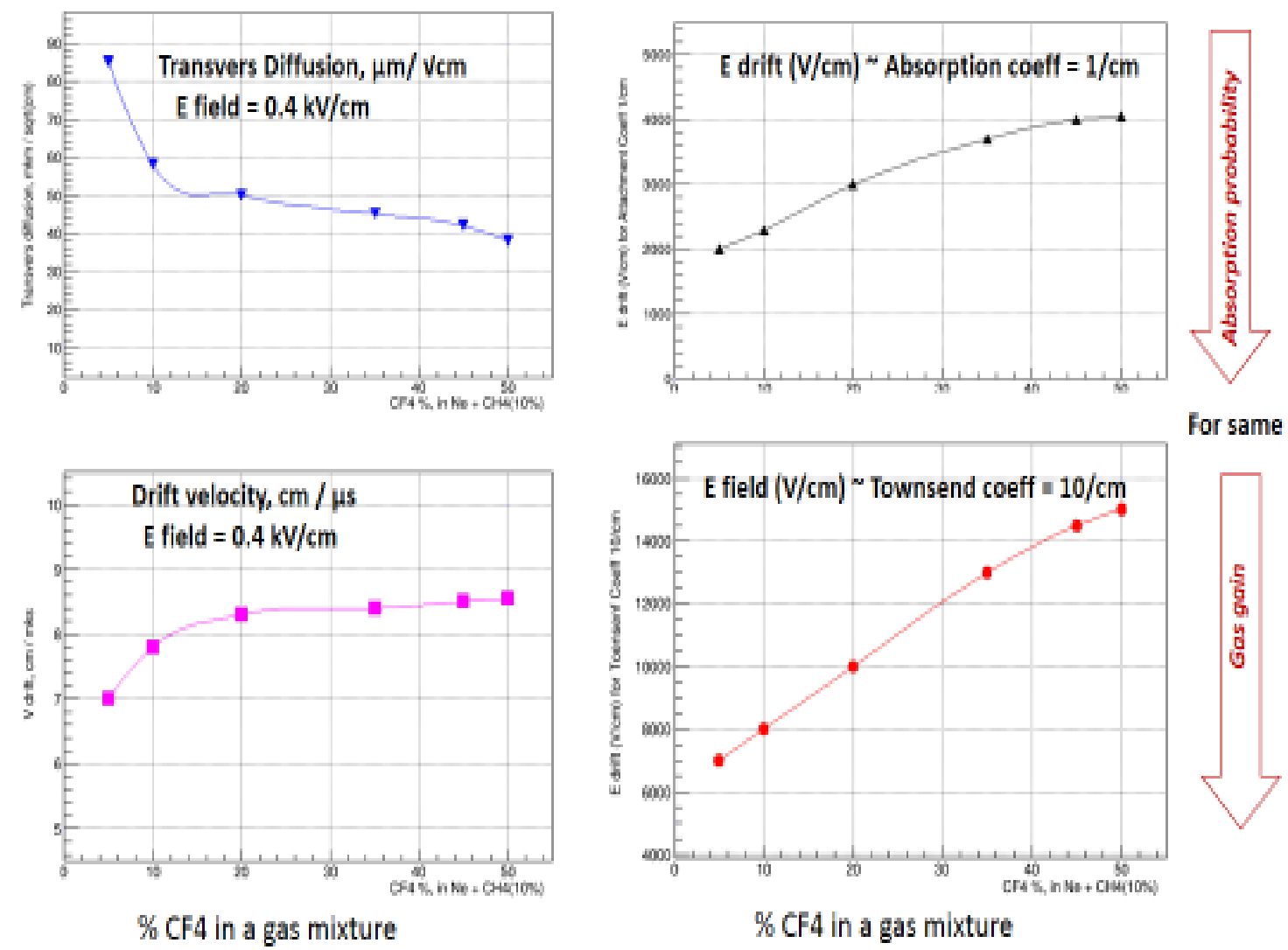

For same E

$\%$ CF4 in a gas mixture

$\%$ CF4 in a gas mixture

[3] Prakhar Garg, private communication, http://skipper.physics.sunysb.edu/ prakhar/tpc/HTML_Gases/split.h tml 


\section{Gain Structure Options to Minimize IBF}

Layered MPGD amplification stages provide a means to control the flow of charge by tuning the electric field in several drift gaps

Drift Volume

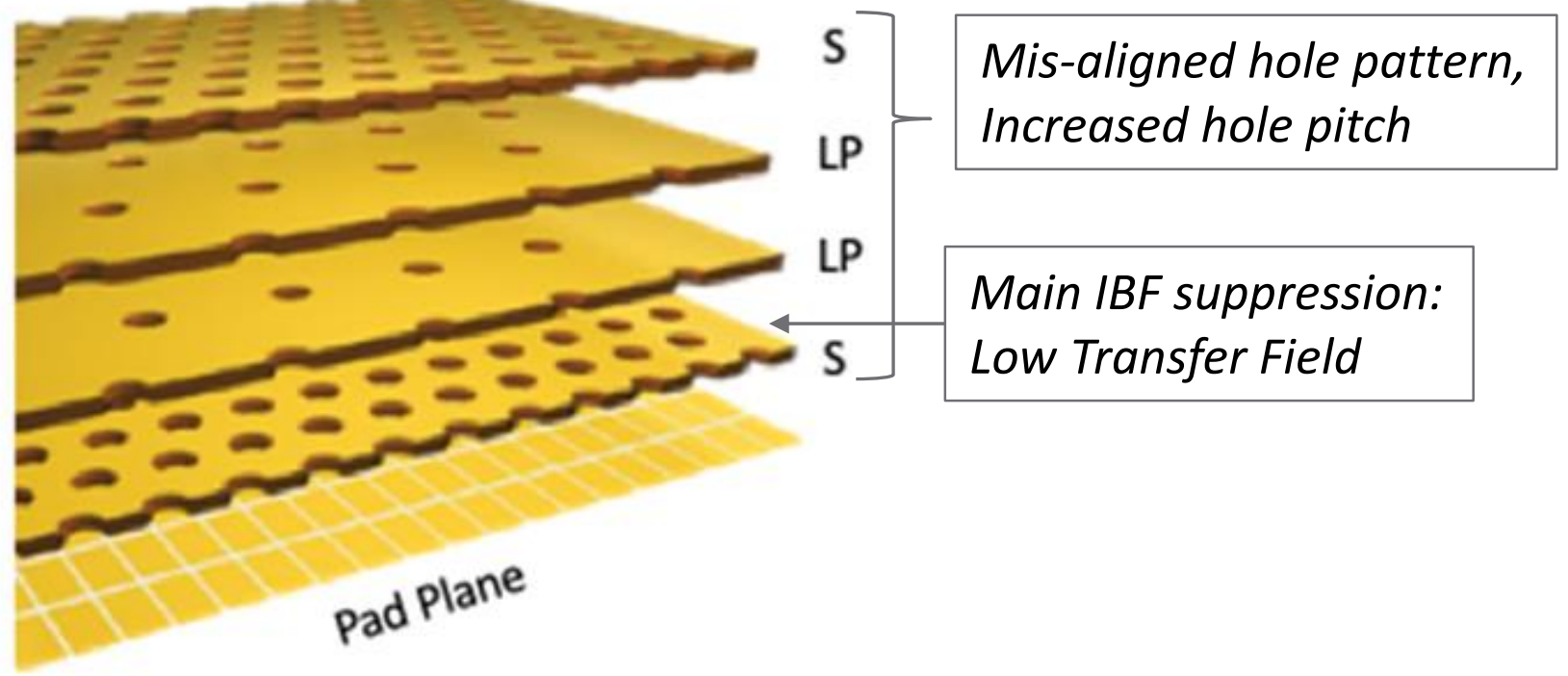

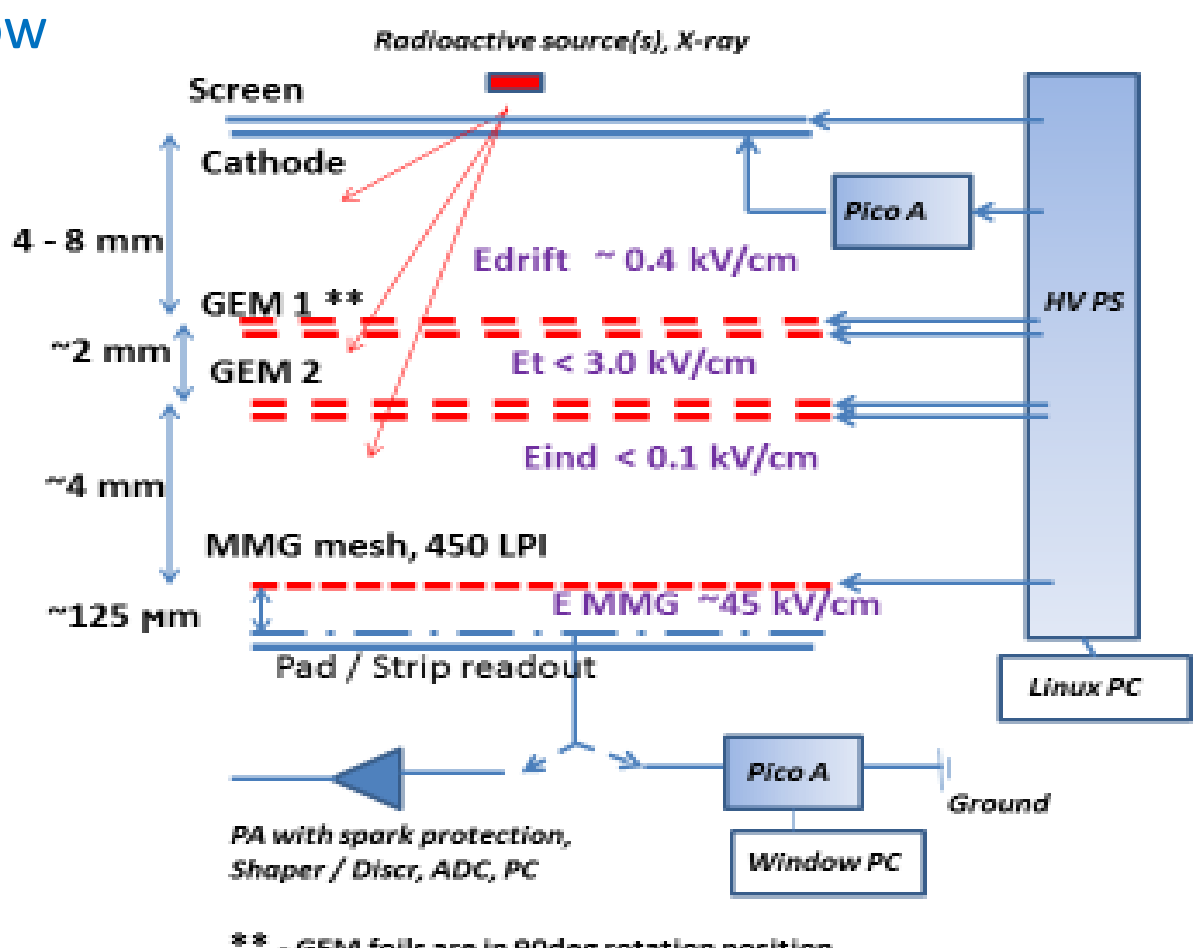

**. GEM foils are in 90deg rotation position
- A 4GEM ion blocking scheme was realized by the ALICE TPC upgrade project using 4 unique GEM foils

- The SPHENIX TPC will utilize a similar concept with some essential modifications

- Top GEM Gain =1 ( Zero fluctuations, $\Delta \mathrm{E} / \mathrm{E}$ undamaged)

- Sacrifice some electron transparency at lower gain stages to improve IBF
- A novel hybrid MMG + 2GEM scheme was proposed by the Yale University team as an alternative to the ALICE concept

- Fully exploits large $E_{\text {drift }} / E_{\text {induction }}$ field ratio of MMG to strongly suppress IBF

- Small GEM gain (low IBF): GEMs effectively transfer primary charge and simultaneously act as effective IBF shield 


\section{IBF Comparison w/ ALICE Gas Mixture}

MMG is the primary gas gain element with a maximum field ratio between the amplification and induction gaps, resulting in a MMG IBF value of $\sim 1 \%$ or lower

- The voltage on the top GEM assures a continuous drift field in the TPC volume and provides small effective gain to preserve electron statistics for good energy resolution

- The extraction field (between GEMs) can be varied without destroying other parameters (easy tuning)

- The second GEM transfers electron clusters from the first gain step (a high E-field) to the MMG induction gap (a very low E-field), with effective gain for GEM2 is less than one

- Other possible parameters are different pitches and/or patterns of the GEM holes.

All critical TPC parameters of the 4GEM setup can be obtained for the MMG + 2 GEMs option, with even lower IBF by a factor $2-2.5$ and smaller max voltage

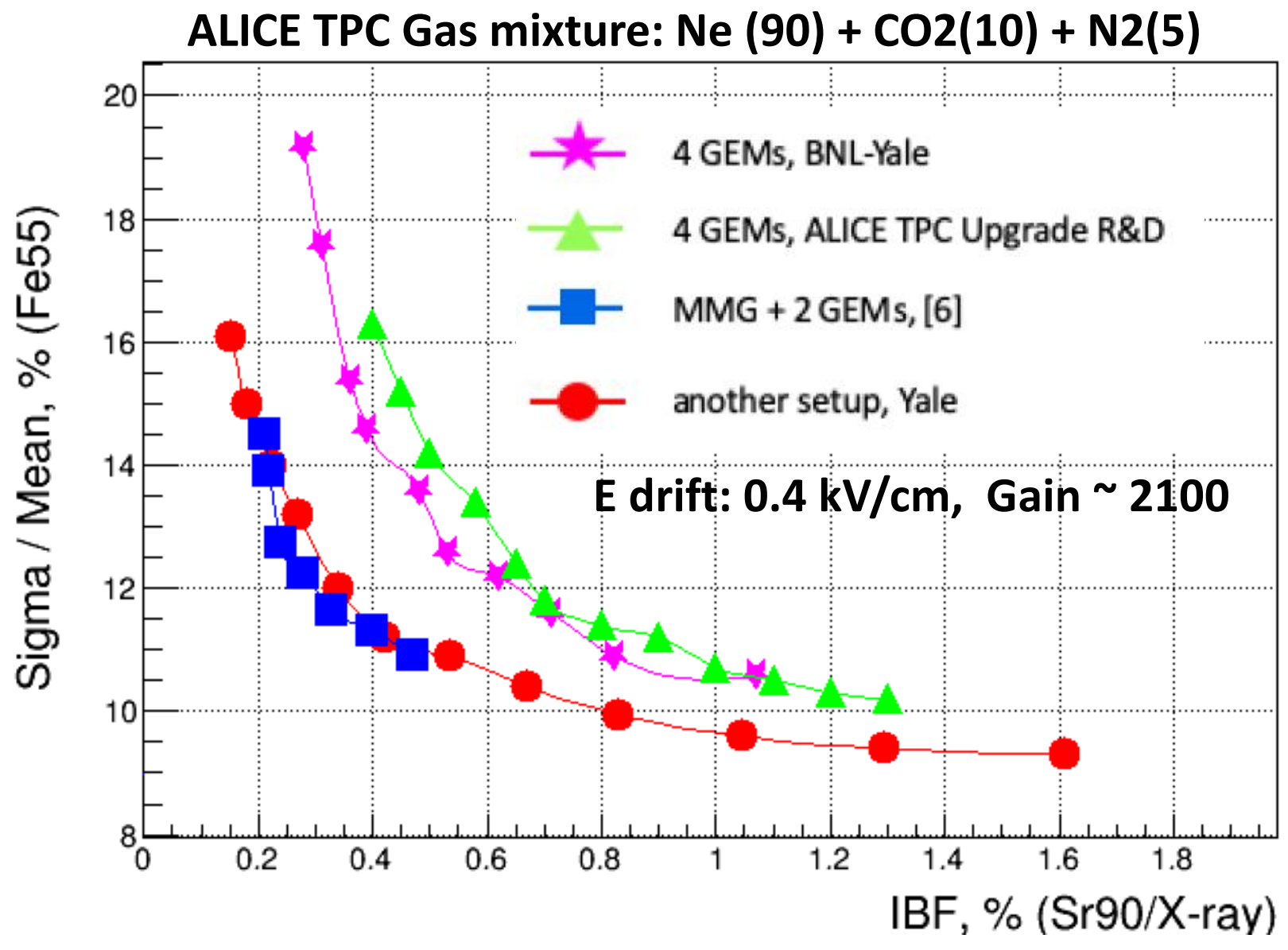

[6] S. Aiola et al, NIM 834 (2016) 149 


\section{Two Gain Options for EIC TPC}

MMG + 2 GEMs: $\mathrm{Ne}+\mathrm{CF} 4+\mathrm{CH} 4$ gas mixtures

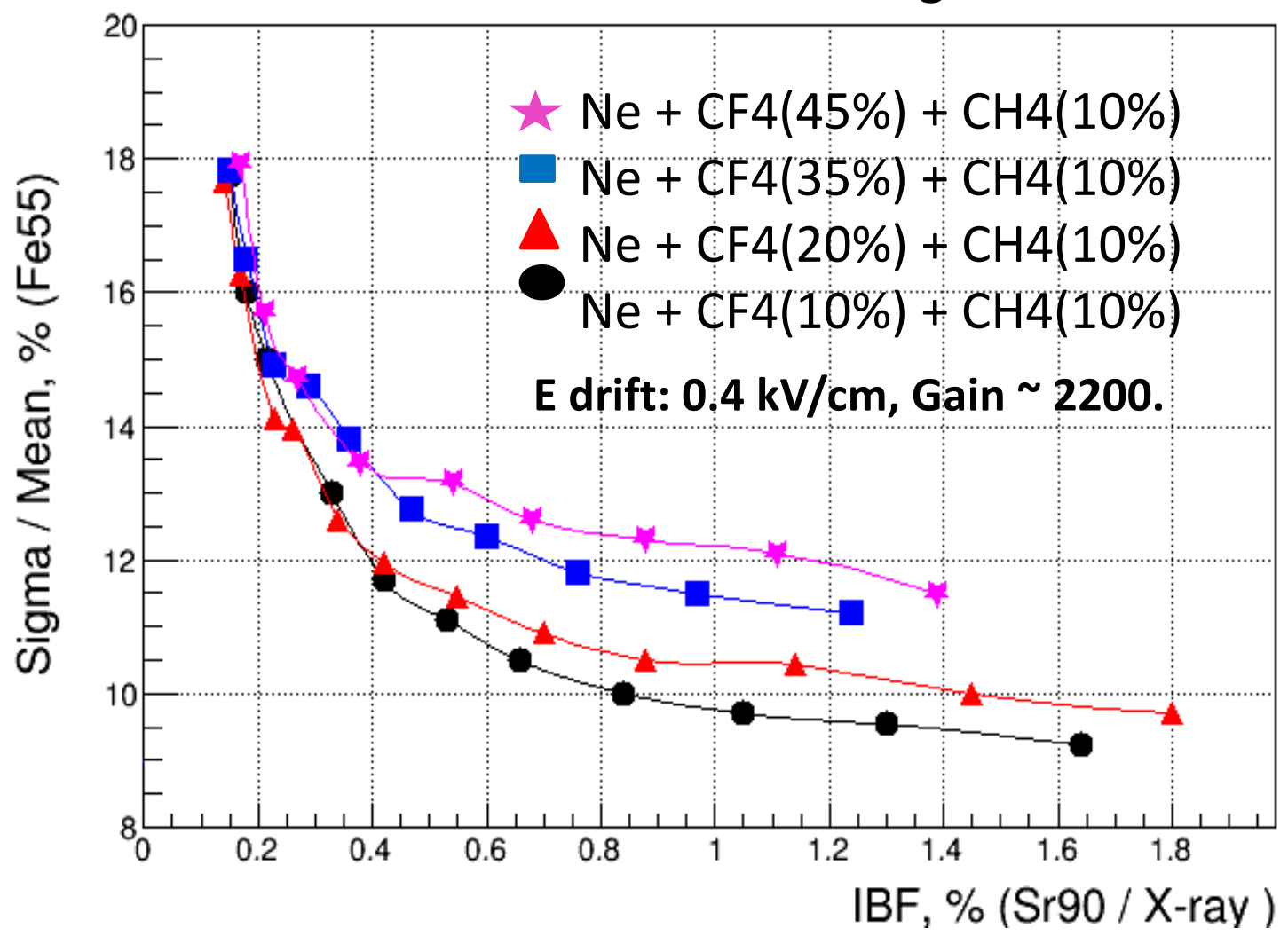

ALICE TPC 4GEM upgrade: Ne + CF4 + CH4 mixtures

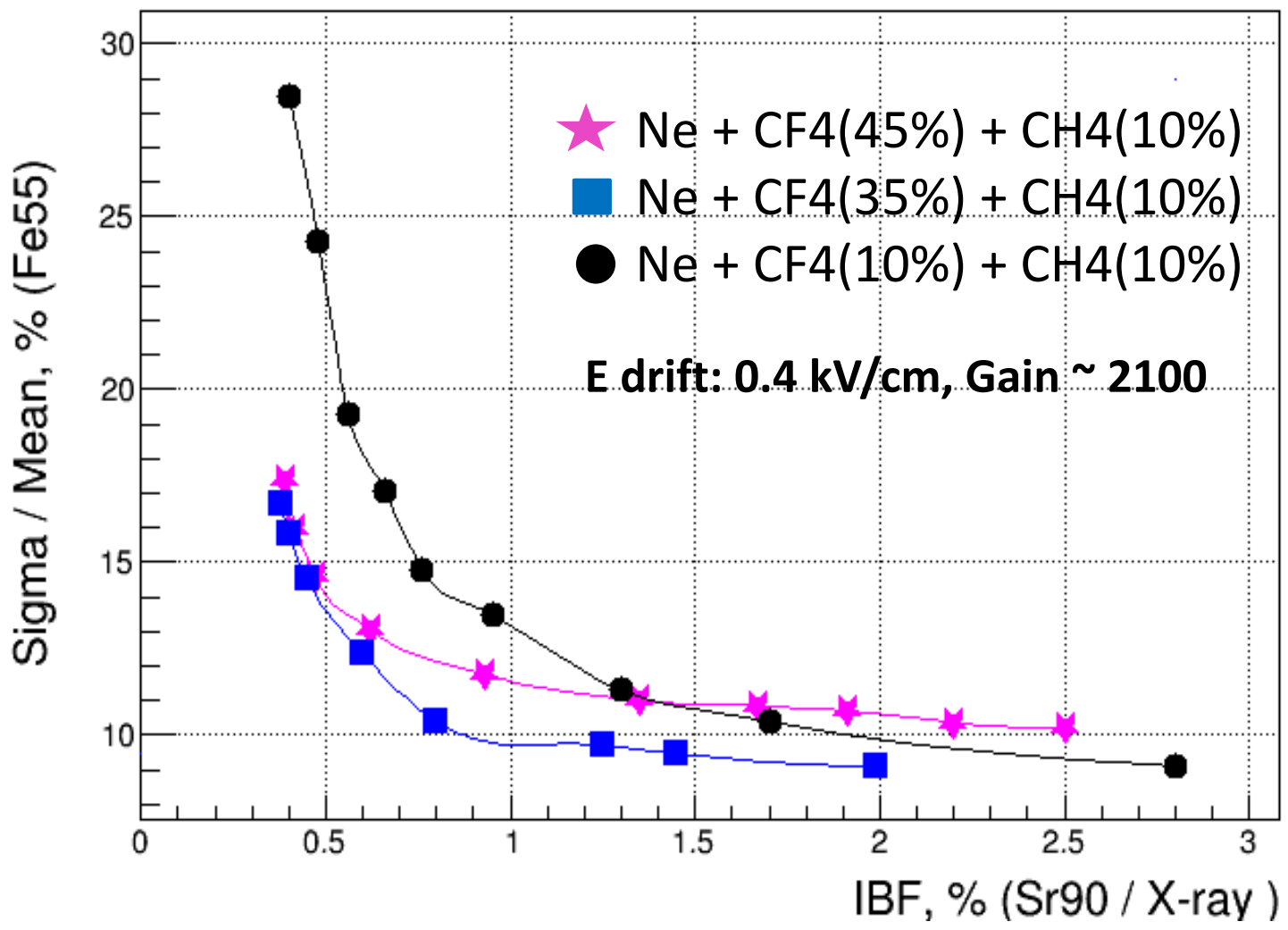

For the $\mathrm{MMG}+2 \mathrm{GEM}$ option 10 percent of $\mathrm{CF}_{4}$ provides the best performance in terms of energy resolution and IBF, while for the 4GEM option 35 - 40\% performs best.

- Best IBF results for $12 \%$ E. Res: $0.40 \%$ (MMG + 2 GEMs) and 0.60\% (4 GEMs)

- Best IBF results for $16 \%$ E. Res: $0.18 \%$ (MMG +2 GEMs) and $0.40 \%$ (4 GEMs) 


\section{Stability Tests with $\mathrm{x}$-ray Source}

MMG + 2GEM exposed to high rate of $x-$ rays with anode current reaching $10 \mathrm{nA} / \mathrm{cm}^{2}$

- Similar probability of MMG sparking that could occur when high momentum particles interact in the mesh material

- Stability test performed at 2 different MMG potentials: $450 \mathrm{~V}$ and $480 \mathrm{~V}$

- Stable operation: 7 hrs of running with no spark!

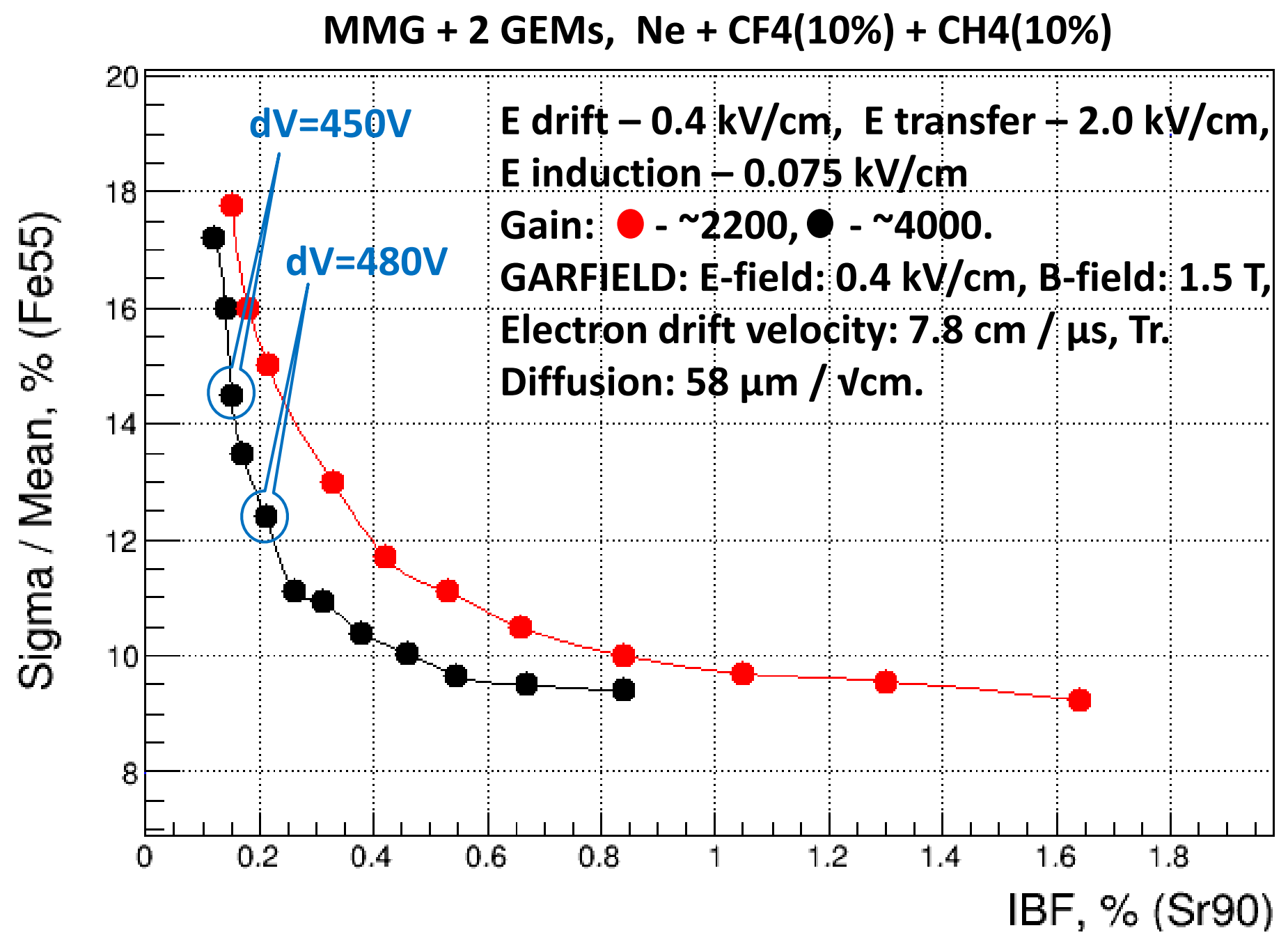




\section{Resistive Layer Protection}

In the event of a spark, a resistive protection layer on the $r / o$ reduces the induced voltage drop and dead time

- $10 \times 10 \mathrm{~cm}^{2} \mathrm{MMG}+4 \times 7.5 \mathrm{~mm}^{2}$ pad r/o

- Spark trigger rate $\sim 1 / 20 \mathrm{~s}$

- Signal from divider connected to MMG mesh

- Signals recorded on oscilloscope

\section{Results:}

- No Protection Layer

- HV drop = 30V (significant gain drop)

- $630 \mu$ s recovery time

- With Resistive Layer: $1 \mathrm{M} \Omega / \mathrm{cm}^{2}$

- HV drop 0.4V (negligible effect)

- $600 \mu \mathrm{s}$ recovery time
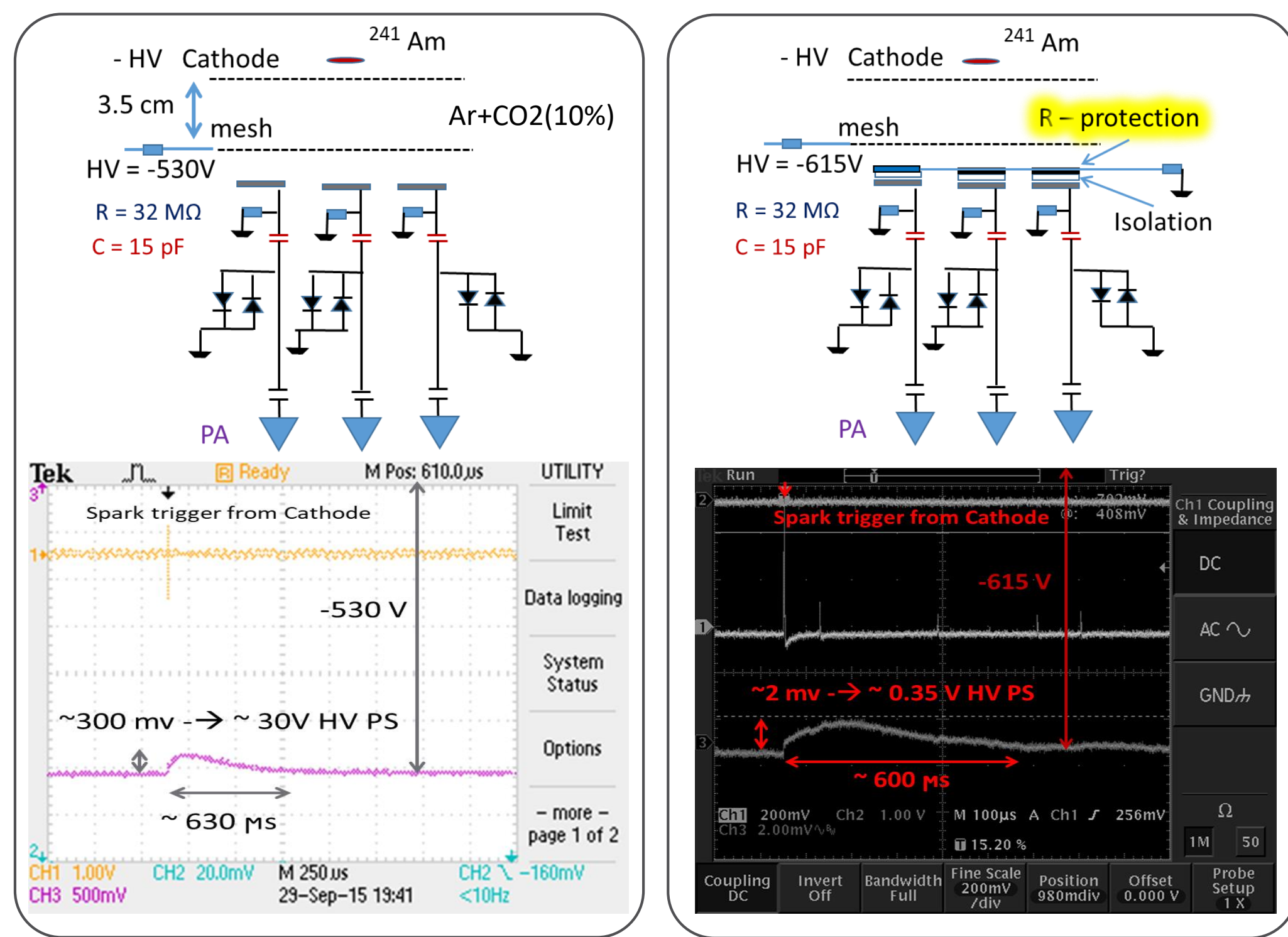


\section{Low Mass Readout Options for TPC}

In order to measure the outgoing electron in EIC collisions with high precision momentum and tracking, a dense readout at the TPC endcap (for $|\eta|>1$ ) must be avoided

Possible solutions

A. Si-based readout: TimePix with $55 \times 55 \mu \mathrm{m}^{2}$ pixel (demonstrated with 3 GEM or Ingrid gain options by ILC team)

- Large sampling of primary ionization electrons with excellent $\mathrm{dE} / \mathrm{dx}$ performance

- Data flow/volume can potentially be challenging, but for EIC events with relatively few tracks per event, this may not be an issue

B. Couple charge from large $\left(\sim 1 \times 10 \mathrm{~mm}^{2}\right)$ anode pads of TPC $\mathrm{r} / \mathrm{o}$ plane to input of high density channels of Si readout

- Additional CCD element may be needed to facilitate the transfer of charge

C. Reposition TPC endcap readout in the electron-going direction to dramatically minimize material thickness for $|\eta|>1$

- Split TPC into two halves: one half with cathode near IP, one standard

\section{Central Field Cage Cathode}

MPGD R/O + Services

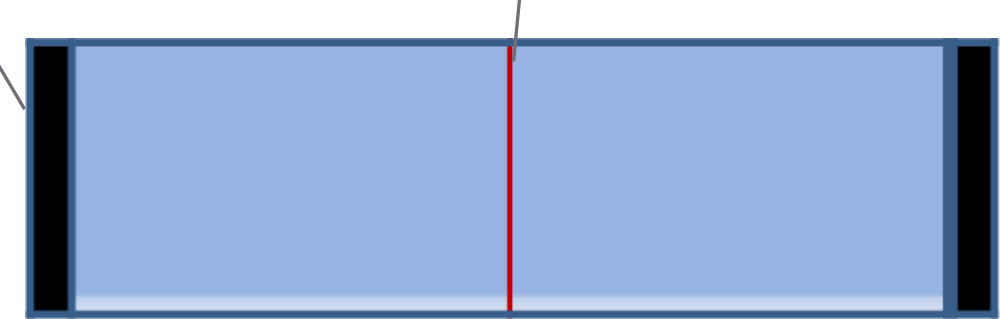

Ion e-

\section{Dual Cathodes}

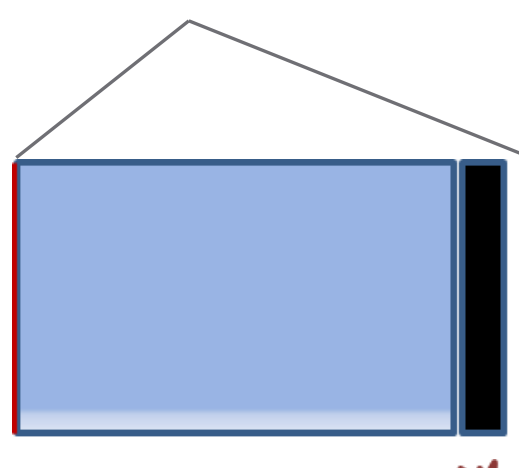

Ion
- Challenges

- This concept needs to be tested with simulation

- Rapidity hole near $\eta=0$

- Impact on Physics seems small (Jets, Abs. X-section measurements?)

- Acceptance of vertex det. obscured

Routing of services, cabling 


\section{Summary}

Investigated crucial parameters for a future high rate TPC at the EIC: ion back flow, energy resolution $(\mathrm{dE} / \mathrm{dx})$, electron and ion drift speed, electron diffusion, and stability

Concentrated on two MPGD gain structures: 4GEM and MMG+2GEM

- For the hybrid option, we achieved an IBF below $0.3 \%$ and an $\Delta \mathrm{E} / \mathrm{E}<12 \%$ for $\mathrm{Fe}^{55} \mathrm{x}$-rays at a gain of 2000 for several gas mixtures

- Optimal gas mixture ratios were identified for both the 4GEM and hybrid option

- We have demonstrated the stability of operation for the hybrid gain element with the optimal working gas

- We have also showed that for relatively rare spark events, the impact on the performance of the hybrid gain element is negligible

Conclusion: The hybrid amplification stage allows for a TPC design that can operate in a continuous mode, serves as a viable option to limit space charge distortions in high-rate TPCs, and guarantees that $\mathrm{dE} / \mathrm{dx}$, ionization cluster space reconstruction resolution, drift parameters and detector stability will not be compromised 